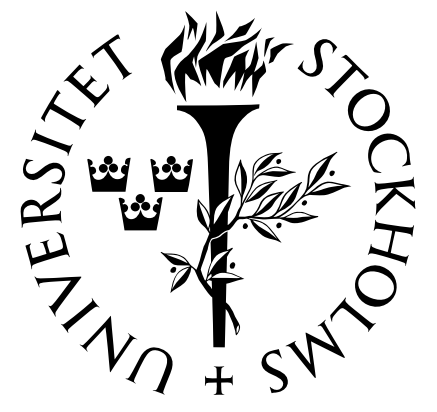

\title{
On the spectral gap for networks of beams
}

\author{
Pavel Kurasov \\ Jacob Muller
}

Research Reports in Mathematics

Number 2, 2020

Department of Mathematics

STOCKHOLM UNIVERSITY 
Date of publication: 17 January, 2020.

2010 Mathematics Subject Classification:

Primary 34L15, 81U40;

Secondary 34L40, 74K10, 81V99.

Keywords: Almost periodic functions, quantum graphs.

Postal address:

Department of Mathematics

Stockholm University

SE-106 91 Stockholm

Sweden

Electronic addresses:

http://www.math.su.se

info@math.su.se 


\begin{abstract}
A notion of standard vertex conditions for beam operarators (the fourth derivative) on metric graphs is presented, and the spectral gap (the difference between the first two eigenvalues) for the operator with these conditions is studied. Upper and lower estimates for the spectral gap are obtained, and it is shown that stronger estimates can be obtained for certain classes of graphs. Graph surgery is used as a technique for estimation. A geometric version of the Ambartsumian theorem for networks of beams is proved.
\end{abstract}

\title{
1. INTRODUCTION
}

In this paper we discuss the problem of estimating the spectral gap for beam operators. These are operators on metric graphs with differential expression $\frac{\mathrm{d}^{4}}{\mathrm{~d} x^{2}}$ on a certain domain of functions satisfying in addition vertex conditions. We focus on a particular set of vertex conditions, which we call standard, which can be seen as an analogue of the standard vertex conditions for quantum graphs. There are a number of possible candidates for the standard beam operator conditions (see for instance $[5,6]$ ), but we opt for the choice which corresponds to the positive operator with the largest spectral gap.

Some estimates for the spectral gap of the standard beam operator are found by minimisation of the Rayleigh quotient. We also extend the notion of graph surgery for quantum graphs $[1,4,7]$ to beam operators. In this approach, one uses topological alterations of graphs affecting the eigenvalues in a predictable manner, and this leads to explicit spectral estimates. With these techniques, certain classes of metric graphs permit stronger upper estimates (bipartite graphs), whilst others permit stronger lower estimates (Eulerian graphs).

Studies of properties of beam operators with other specific vertex conditions include [3], and spectral asymptotics for general vertex conditions are investigated in [8].

Beam operators correspond to physical networks of beams connected together in some way. Eigenstates of the beam operator represent stationary states of the systems, with the functions themselves describing the transverse displacement of points along the beam (see [9] for more details). The problem of maximising the spectral gap for beam operators also has the significant practical interpretation of increasing the gap 
between resonant frequencies in a physical system. This may be useful in the context of networks of beams where occurrence of resonant frequencies may have a detrimental impact (for instance Harrington rods for scoliosis).

\section{THE OPERATOR}

Let $\Gamma$ be a compact finite connected metric graph with edge set $\mathbf{E}$ (consisting of $N$ edges $E_{1}, \ldots, E_{N}$, identified as intervals from different copies of $\mathbb{R}$ ) and vertex set $\mathbf{V}$ (where the vertices $V_{m} \in \mathbf{V}$ are identified with disjoint subsets of the set $\left\{x_{1}, \ldots, x_{2 N}\right\}$ of endpoints of the edges). We do not require that the metric graph $\Gamma$ can be embedded in $\mathbb{R}^{n}$.

A beam operator on $\Gamma$ is an operator $A(\Gamma)$ in $L_{2}(\Gamma)$ with differential expression $\frac{\mathrm{d}^{4}}{\mathrm{~d} x^{4}}$ acting on functions from $W_{2}^{4}(\Gamma \backslash \mathbf{V})$ satisfying certain vertex conditions. These vertex conditions should not mix between vertices, i.e. the boundary terms at endpoints in one vertex should not depend on those of endpoints in another vertex.

Many different vertex conditions can be considered (see [5]), but we are looking for an analogue of standard vertex conditions ${ }^{1}$ introduced for the Laplacian (second derivative operator) at every vertex $V_{m} \in \mathbf{V}$ :

$$
\left\{\begin{array}{l}
u \text { is continuous at } V_{m} \\
\sum_{x_{j} \in V_{m}} \partial u\left(x_{j}\right)=0
\end{array}\right.
$$

where the sum is taken over all endpoints joined at the vertex and $\partial u$ denote the normal derivatives. The best way to justify the standard conditions (2.1) is to consider the Dirichlet form $\int_{\Gamma}\left\|u^{\prime}\right\|^{2} \mathrm{~d} x$ defined on the functions from $W_{2}^{1}(\Gamma \backslash \mathbf{V})$ which are assumed in addition to be continuous at the vertices. This form is closed and bounded from below. The corresponding operator is $L^{\mathrm{st}}(\Gamma)=-\frac{\mathrm{d}^{2}}{\mathrm{~d} x^{2}}$ defined on the functions from $W_{2}^{2}(\Gamma \backslash \mathbf{V})$ satisfying the standard conditions (2.1) at every vertex.

The formal quadratic form of the (positive) beam operator is given by

$$
a(u, u)=\left\|u^{\prime \prime}\right\|^{2}=\int_{\Gamma}\left|u^{\prime \prime}\right|^{2} \mathrm{~d} x .
$$

with $u \in W_{2}^{2}(\Gamma \backslash \mathbf{V})$. One may impose vertex conditions on functions in the domain of this form which then corresponds to a certain positive beam operator. The spectrum of this operator is purely discrete and

\footnotetext{
${ }^{1}$ Standard conditions are also called Kirchhoff, Neumann or natural by some authors.
} 
non-negative, since each operator is a finite rank perturbation (in the resolvent sense) of the operators on single edges with any self-adjoint boundary conditions (see $[2,8]$ ). If no vertex conditions are assumed, then the corresponding operator $A(\Gamma)$ is just $\frac{\mathrm{d}^{4}}{\mathrm{~d} x^{4}}$ defined on the functions satisfying the following conditions at all endpoints $x_{j}$ of the edges:

$$
\left\{\begin{array}{l}
\partial^{2} u\left(x_{j}\right)=0 \\
\partial^{3} u\left(x_{j}\right)=0
\end{array}\right.
$$

It is clear that in this case $A(\Gamma)$ is just an orthogonal sum of similar operators on the edges $E_{n}$ :

$$
A(\Gamma)=\oplus_{n=1}^{N} A\left(E_{n}\right) .
$$

This operator does not correspond to the graph $\Gamma$, but rather to a collection of non-connected edges.

In order to produce a model where the edges are connected, one has to introduce vertex conditions on functions in the domain of the quadratic form. It is natural to enforce the continuity condition

$$
u\left(x_{i}\right)=u\left(x_{j}\right), \text { provided } x_{i}, x_{j} \in V_{m} .
$$

This condition means that the beams are touching at the vertices. If no further conditions are imposed on the values of the functions at the endpoints (i.e. any further conditions concern only the derivatives) then certainly the constant function $\psi_{1}(x) \equiv 1$ lies in the domain of the quadratic form and hence also in the domain of the corresponding positive operator $A(\Gamma)$. Consequently the first eigenvalue of $A(\Gamma)$ is zero, and the spectral gap - the difference between the first two eigenvalues — is simply equal to the second eigenvalue:

$$
\lambda_{2}(A(\Gamma))-\lambda_{1}(A(\Gamma))=\lambda_{2}(A(\Gamma)) .
$$

In applications, one may be interested in maximising the distance between eigenvalues. A good starting point would be to maximise the spectral gap. In what follows we are going to consider the quadratic form (2.2) defined on the functions from $W_{2}^{2}(\Gamma \backslash \mathbf{V})$ satisfying the following conditions:

$$
\left\{\begin{array}{l}
u\left(x_{i}\right)=u\left(x_{j}\right), \text { provided } x_{i}, x_{j} \in V_{m} ; \\
\partial u\left(x_{j}\right)=0 .
\end{array}\right.
$$

The corresponding operator $A^{\mathrm{st}}(\Gamma)=\frac{\mathrm{d}^{4}}{\mathrm{~d} x^{4}}$ is defined on the domain of functions from $W_{2}^{4}(\Gamma \backslash \mathbf{V})$ satisfying the vertex conditions

$$
\left\{\begin{array}{l}
u\left(x_{i}\right)=u\left(x_{j}\right), \text { provided } x_{i}, x_{j} \in V_{m} \\
\partial u\left(x_{j}\right)=0 \\
\sum_{x_{j} \in V_{m}} \partial^{3} u\left(x_{j}\right)=0
\end{array}\right.
$$


at every vertex $V_{m}$.

Definition. The operator $A^{\mathrm{st}}(\Gamma)=\frac{\mathrm{d}^{4}}{\mathrm{~d} x^{4}}$ with domain equal to the set of functions from $W_{2}^{4}(\Gamma \backslash \mathbf{V})$ satisfying the vertex conditions (2.6) is called the standard beam operator. We refer to these vertex conditions are called standard.

This operator is uniquely determined by the graph $\Gamma$. One may refer to its spectrum as the spectrum of the graph.

\section{On Spectral Properties of $A^{\text {st }}(\Gamma)$.}

The standard beam operator $A^{\text {st }}(\Gamma)$ has the smallest quadratic form domain among those obtained by imposing vertex conditions on functions in $W_{2}^{2}(\Gamma)$; the eigenvalues (and in particular the spectral gap) of the corresponding positive operator $\frac{\mathrm{d}^{4}}{\mathrm{~d} x^{4}}$ are the largest possible as is clear from the following well-known result:

Proposition 1. Let $A$ be a self-adjoint operator with quadratic form a, semi-bounded from below, with discrete spectrum. The eigenvalues $\lambda_{j}(A)$ of A satisfy

$$
\begin{aligned}
\lambda_{j}(A) & =\min _{\substack{\mathcal{V}_{j} \subset \operatorname{dom}(a), \operatorname{dim}\left(\mathcal{V}_{j}\right)=j}} \max _{u \in \mathcal{V}_{j}} \frac{a(u, u)}{\|u\|^{2}} \\
= & \max _{\substack{\mathcal{V}_{j-1} \subset \operatorname{dom}(a), \operatorname{dim}\left(\mathcal{V}_{j-1}\right)=j-1}} \min _{u \in \mathcal{V}_{j-1}^{\perp}} \frac{a(u, u)}{\|u\|^{2}} .
\end{aligned}
$$

In particular, given a compact finite connected graph $\Gamma$, the spectral gap of $A^{\text {st }}(\Gamma)$ can be calculated as

$$
\lambda_{2}\left(A^{\mathrm{st}}(\Gamma)\right)=\min _{\substack{u \in \operatorname{dom}(a), \int_{\Gamma} u \mathrm{~d} x=0}} \frac{\int_{\Gamma}\left|u^{\prime \prime}\right|^{2} \mathrm{~d} x}{\int_{\Gamma}|u|^{2} \mathrm{~d} x},
$$

where now a is the quadratic form for $A^{\mathrm{st}}(\Gamma)$.

The operator $A^{\text {st }}(\Gamma)$ is in fact the Friedrichs extension of the symmetric beam operator with conditions (2.3) and (2.5) imposed simultaneously, as discussed in [5].

It has already been mentioned that $\psi_{1}(x) \equiv 1$ is a ground state; if the graph is connected then the ground state is unique. To see this directly, 
assume that $u$ is an eigenfunction corresponding to the eigenvalue $\lambda=0$, then

$$
u^{(4)}(x)=0 \Rightarrow u(x)=a_{n}+b_{n} x+c_{n} x^{2}+d_{n} x^{3}, x \in E_{n},
$$

for each edge $E_{n}$. This function also minimises the Rayleigh quotient (3.1), which means that it satisfies $\int_{\Gamma}\left\|u^{\prime \prime}\right\|^{2} \mathrm{~d} x=0$, and thus

$$
u^{\prime \prime}(x)=0 \Rightarrow c_{n}=d_{n}=0 .
$$

The function $u(x)=a_{n}+b_{n} x$ satisfies the Neumann condition $\partial u\left(x_{j}\right)=0$ at each endpoint $x_{j}$ if and only if $b_{n}=0$. The continuity condition at the vertices implies that all $a_{n}$ are equal, i.e. that the function $\psi$ is constant on the whole graph since graph is connected.

One can get some simple upper bounds for the spectral gap by applying Proposition 1.

Lemma 2. Let $\Gamma$ be a compact finite connected metric graph with total length $\mathcal{L}(\Gamma)$ and let $\ell_{n}$ be the length of the edge $E_{n}$ for each $n$. Then

$$
\lambda_{2}\left(A^{\mathrm{st}}(\Gamma)\right) \leq 16\left(\frac{\pi}{\mathcal{L}(\Gamma)}\right)^{4} \sum_{n=1}^{N}\left(\frac{\mathcal{L}(\Gamma)}{\ell_{n}}\right)^{3} .
$$

If in particular $\Gamma$ is a bipartite graph, then

$$
\lambda_{2}\left(A^{\mathrm{st}}(\Gamma)\right) \leq\left(\frac{\pi}{\mathcal{L}(\Gamma)}\right)^{4} \sum_{n=1}^{N}\left(\frac{\mathcal{L}(\Gamma)}{\ell_{n}}\right)^{3} .
$$

Proof. Let $u$ be the function, in the domain of the quadratic form of $A^{\text {st }}(\Gamma)$, defined by $u(x)=\cos \left(\frac{2 \pi x}{\ell_{n}}\right)$ on each edge $E_{n}=\left[0, \ell_{n}\right]$. Then

$$
\frac{\int_{\Gamma}\left|u^{\prime \prime}\right|^{2} \mathrm{~d} x}{\int_{\Gamma}|u|^{2} \mathrm{~d} x}=\frac{\sum_{n} \frac{8 \pi^{4}}{\ell_{n}^{3}}}{\sum_{n} \frac{\ell_{n}}{2}}=16\left(\frac{\pi}{\mathcal{L}(\Gamma)}\right)^{4} \sum_{n=1}^{N}\left(\frac{\mathcal{L}(\Gamma)}{\ell_{n}}\right)^{3} .
$$

Since clearly $\langle 1, u\rangle=\int_{\Gamma} u(x) \mathrm{d} x=0$, the estimate (3.4) follows from Proposition 1.

If $\Gamma$ is bipartite, then applying the same approach to the function $u$ defined by $u(x)=\cos \left(\frac{\pi x}{\ell_{n}}\right)$ on $E_{n}$ (parameterised in the appropriate direction such that $u$ is continuous on $\Gamma$ ) gives the second estimate (3.5).

The estimates from Lemma 2 are quite bad if $\Gamma$ contains some edges which are much shorter than the others. In that case better estimates may be found, for instance by comparison with eigenvalues of other beam operators. 
Let $A^{\mathrm{F}}(\Gamma)$ be the beam operator defined on functions from $W_{2}^{4}(\Gamma \backslash \mathbf{V})$ satisfying the vertex conditions

$$
\left\{\begin{aligned}
u\left(x_{j}\right) & =0 \\
\partial u\left(x_{j}\right) & =0
\end{aligned}\right.
$$

at every endpoint $x_{j}$. This is the Friedrichs extension of the minimal symmetric beam operator whose domain consists only of functions from $W_{2}^{4}(\Gamma \backslash \mathbf{V})$ satisfying (3.6) and (2.3) simultaneously. Since $A^{\text {st }}(\Gamma)$ is also a self-adjoint extension of this minimal operator, it follows that $\lambda_{k}\left(A^{\text {st }}(\Gamma)\right) \leq$ $\lambda_{k}\left(A^{\mathrm{F}}(\Gamma)\right)$ for all $k$. Observe that the smallest eigenvalue of $A^{\mathrm{F}}(\Gamma)$ is strictly positive: the eigenvalues of $A^{\mathrm{F}}(\Gamma)$ are in fact the eigenvalues of $A^{\mathrm{F}}\left(E_{n}\right)$ for each edge $E_{n}$. Thus $\lambda_{1}\left(A^{\mathrm{F}}(\Gamma)\right) \leq \lambda_{1}\left(A^{\mathrm{F}}\left(E_{1}\right)\right)$ with equality if $E_{1}$ is the longest edge. Solutions of $\frac{\mathrm{d}^{4}}{\mathrm{~d} x^{4}} \psi=\lambda \psi$ for $\lambda=k^{4}>0$ are of the form $\psi(x)=a \mathrm{e}^{\mathrm{i} k x}+b \mathrm{e}^{-\mathrm{i} k x}+c \mathrm{e}^{-k x}+d \mathrm{e}^{k x}$. By imposing the vertex conditions of $A^{\mathrm{F}}\left(E_{1}\right)$, one sees that $\lambda=k^{4}$ is an eigenvalue if and only if $x=k \ell_{1}$ is a positive root of

$$
\operatorname{det}\left(\begin{array}{cc}
\sin x-\cos x+\mathrm{e}^{-x} & -\sin x-\cos x+\mathrm{e}^{x} \\
\sin x+\cos x-\mathrm{e}^{-x} & \sin x-\cos x+\mathrm{e}^{x}
\end{array}\right) \equiv 4(1-\cos x \cosh x) .
$$

Let $\mu>0$ be such that $x=\pi \mu$ is the smallest positive root. By numerical approximation, one finds that $\mu \lesssim 1.506$. Thus

$$
\lambda_{1}\left(A^{\mathrm{F}}\left(E_{1}\right)\right) \leq \mu^{4}\left(\frac{\pi}{\ell_{1}}\right)^{4} .
$$

Let $\psi_{1}$ be the corresponding eigenfunction of $A^{\mathrm{F}}\left(E_{1}\right)$. It can be extended to a function $\phi$ in the domain of the quadratic form of the standard beam operator $A^{\text {st }}(\Gamma)$ such that $\langle 1, \phi\rangle=0$ as follows:

$$
\phi(x)= \begin{cases}\psi_{1}(x) & \text { if } x \in E_{1}, \\ -\frac{\ell_{1}}{\ell_{2}} \psi_{1}\left(\frac{\ell_{1} x}{\ell_{2}}\right) & \text { if } x \in E_{2} \\ 0 & \text { otherwise }\end{cases}
$$

Here we have assumed that $E_{1}=\left[0, \ell_{1}\right]$ and $E_{2}=\left[0, \ell_{2}\right]$ without loss of generality. Then

$$
\frac{\int_{\Gamma}\left|\phi^{\prime \prime}\right|^{2} \mathrm{~d} x}{\int_{\Gamma}|\phi|^{2} \mathrm{~d} x}=\frac{1+\left(\frac{\ell_{1}}{\ell_{2}}\right)^{5}}{1+\frac{\ell_{1}}{\ell_{2}}} \frac{\int_{E_{1}}\left|\psi_{1}^{\prime \prime}\right|^{2} \mathrm{~d} x}{\int_{E_{1}}\left|\psi_{1}\right|^{2} \mathrm{~d} x}=\frac{1+\left(\frac{\ell_{1}}{\ell_{2}}\right)^{5}}{1+\frac{\ell_{1}}{\ell_{2}}} \lambda_{1}\left(A^{\mathrm{F}}\left(E_{1}\right)\right),
$$

(with the last equality by (3.1)), and so by Proposition 1,

$$
\lambda_{2}\left(A^{\mathrm{F}}(\Gamma)\right) \leq \mu^{4} \pi^{4}\left(\frac{1}{\ell_{1}^{4}}-\frac{1}{\ell_{1}^{3} \ell_{2}}+\frac{1}{\ell_{1}^{2} \ell_{2}^{2}}-\frac{1}{\ell_{1} \ell_{2}^{3}}+\frac{1}{\ell_{2}^{4}}\right) .
$$


For $\ell_{1}+\ell_{2}$ fixed, this estimate is optimal when $\ell_{1}=\ell_{2}$. Assume without loss of generality that $\ell_{1}>\ell_{2}$ (indeed we may as well assume that $\ell_{1}$ is the longest edge). ${ }^{1}$ This estimate depends only on the lengths of two edges, whilst the estimate 3.4 tends to $+\infty$ if one shrinks any of the other edges to 0 .

\section{SURGERY FOR STANDARD BEAM OPERATORS}

Graph surgery is an umbrella term used to describe a number of operations that one can perform on a graph - like cutting through or gluing together vertices, lengthening edges, or adding edges - whose effects on the spectrum of the operator on the graph (for instance increasing or decreasing eigenvalues) are predictable. A detailed overview of such results can be found in $[1,7]$. These techniques can of course be employed for beam operators, and analogous results to those for quantum graphs can be obtained. We present just one of those here, and we shall soon see that it it is convenient for obtaining lower estimates for the spectral gap.

Theorem 3. If the graph $\tilde{\Gamma}$ is obtained from $\Gamma$ by cutting through one or several vertices, then the following inequality holds for the eigenvalues of the standard beam operators:

$$
\lambda_{k}\left(A^{\mathrm{st}}(\Gamma)\right) \geq \lambda_{k}\left(A^{\mathrm{st}}(\tilde{\Gamma})\right), k=1,2, \ldots
$$

Proof. Every function on the graph $\Gamma$ can naturally be considered as a function on $\tilde{\Gamma}$. Moreover, if the function satisfies standard conditions on $\Gamma$, then its image on $\tilde{\Gamma}$ satisfies standard conditions on $\tilde{\Gamma}$. Hence the set of $n$ dimensional subspaces of the domain of the quadratic form increases when vertices are cut. Inequality (4.1) then follows from (3.1) from Proposition 1.

One may repeat the proofs of several further theorems contained in [1] to obtain additional surgery-related eigenvalue inequalities.

\footnotetext{
${ }^{1}$ Observe that (3.7) is certainly better than the upper estimate $\mu^{4}\left(\frac{\pi}{\ell_{2}}\right)^{4}$ which can be directly seen as the ground state eigenvalue of $A^{\mathrm{F}}\left(E_{2}\right)$ and thus an eigenvalue of $A^{\mathrm{F}}(\Gamma)$ (second or higher).
} 


\section{Estimates FOR THE SPECTRAL GAP}

For standard Laplacians on metric graphs we have the following estimate:

$$
\lambda_{2}\left(L^{\mathrm{st}}(\Gamma)\right) \geq\left(\frac{\pi}{\mathcal{L}(\Gamma)}\right)^{2} .
$$

This estimate can be improved in the case of Eulerian graphs (having even degrees of all vertices):

$$
\lambda_{2}\left(L^{\mathrm{st}}(\Gamma)\right) \geq 4\left(\frac{\pi}{\mathcal{L}(\Gamma)}\right)^{2},
$$

or further for two-connected graphs. These are obtained in [7] using graph surgery principles for quantum graphs. A similar result can be achieved by this method for standard beam operators:

Theorem 4. Let $\Gamma$ be a connected finite compact metric graph with total length $\mathcal{L}(\Gamma)$ and let $A^{\mathrm{st}}(\Gamma)$ be the corresponding standard beam operator described by vertex conditions (2.6). The spectral gap for this operator can be estimated as:

$$
\lambda_{2}\left(A^{\mathrm{st}}(\Gamma)\right) \geq\left(\frac{\pi}{\mathcal{L}(\Gamma)}\right)^{4} .
$$

If the graph $\Gamma$ is Eulerian (i.e. the degrees of all vertices are even), then the above estimate can be improved:

$$
\lambda_{2}\left(A^{\mathrm{st}}(\Gamma)\right) \geq 16\left(\frac{\pi}{\mathcal{L}(\Gamma)}\right)^{4} .
$$

Proof. We follow closely the proof presented for the Laplacian in [7]. Let $\psi_{2}$ be an eigenfunction corresponding to $\lambda_{2}\left(A^{\text {st }}(\Gamma)\right)$. Consider the graph $\Gamma^{2}$ obtained from $\Gamma$ by adding to each edge $E_{n}$ a parallel edge $E_{N+n}$ of the same length. Let $A^{\text {st }}\left(\Gamma^{2}\right)$ be the standard beam operator on $\Gamma^{2}$.

The $\lambda_{2}\left(A^{\text {st }}(\Gamma)\right)$-eigenfunction $\psi_{2}$ can be extended to $\Gamma^{2}$ by assigning the same values on the new parallel edges. Obviously the new function is an eigenfunction of $A^{\text {st }}\left(\Gamma^{2}\right)$ with the same eigenvalue (as it satisfies the vertex conditions on $\Gamma^{2}$ and the differential equation on the edges). It follows that

$$
\lambda_{2}\left(A^{\mathrm{st}}(\Gamma)\right) \geq \lambda_{2}\left(A^{\mathrm{st}}\left(\Gamma^{2}\right)\right) .
$$

The degree of every vertex of graph $\Gamma^{2}$ is even, and so the graph is Eulerian and there exists a closed path $\mathcal{P}_{2 \mathcal{L}}$ coming along every edge in $\Gamma^{2}$ precisely 
once. This path can be seen as a graph formed of $2 N$ edges connected pairwise at $2 N$ vertices, where standard conditions for the beam operator are assumed. One can obtain $\mathcal{P}_{2 \mathcal{L}}$ by cutting through vertices in $\Gamma^{2}$, hence we have

$$
\lambda_{2}\left(A^{\mathrm{st}}\left(\Gamma^{2}\right)\right) \geq \lambda_{2}\left(A^{\mathrm{st}}\left(\mathcal{P}_{2 \mathcal{L}}\right)\right) .
$$

The eigenvalues of $A^{\text {st }}\left(\mathcal{P}_{2 \mathcal{L}}\right)$ are relatively difficult to calculate: since the corresponding eigenfunction does not satisfy the differential equation at the (degree two) vertices, vertex conditions are assumed instead. These conditions on functions $u \in W_{2}^{4}\left(\mathcal{P}_{2 \mathcal{L}}\right)$ are as follows:

- continuity of $u$;

- Neumann condition: $u^{\prime}\left(x_{j}\right)=0$ (a pair of conditions at each vertex);

- continuity condition for the third derivative $u^{\prime \prime \prime}$.

The functions from the domain of the quadratic form satisfy just the first two conditions at every vertex on the path.

Consider the beam operator $A\left(C_{2 \mathcal{L}}\right)$ on the loop $C_{2 \mathcal{L}}$ of length $2 \mathcal{L}$. The loop can be seen as one edge $[0,2 \mathcal{L}]$ with the endpoints identified. The corresponding vertex conditions for functions $u \in W_{2}^{4}\left(\mathcal{C}_{2 \mathcal{L}}\right)$ are:

- continuity of $u$;

- continuity of the first derivative $u^{\prime}$;

- continuity of the second derivative $u^{\prime \prime}$;

- continuity of the third derivative $u^{\prime \prime \prime}$.

The functions from the domain of the quadratic form satisfy just the first two conditions.

Every function satisfying Neumann conditions at a degree two vertex automatically has continuous first derivative there. Hence the domain of the quadratic form for $A\left(C_{2 \mathcal{L}}\right)$ is larger than the domain of the quadratic form of $A^{\text {st }}\left(\mathcal{P}_{2} \mathcal{L}\right)$. The following inequality follows:

$$
\lambda_{2}\left(A^{\text {st }}\left(\mathcal{P}_{2 \mathcal{L}}\right)\right) \geq \lambda_{2}\left(A\left(C_{2 \mathcal{L}}\right)\right) .
$$

The chain of inequalities together with the fact that

$$
\lambda_{2}\left(A\left(C_{2 \mathcal{L}}\right)\right)=\left(\frac{\pi}{\mathcal{L}(\Gamma)}\right)^{4}
$$

implies the lower estimate in (5.3). 
It is enough to notice that if the graph $\Gamma$ is already Eulerian, then there is no necessity to double it building $\Gamma^{2}$. By cutting through vertices the graph $\Gamma$ can be turned into a path $\mathcal{P}_{\mathcal{L}}$ of the length $\mathcal{L}$. Hence we have;

$$
\lambda_{2}\left(A^{\mathrm{st}}(\Gamma)\right) \geq \lambda_{2}\left(\mathcal{P}_{\mathcal{L}}\right) \geq \lambda_{2}\left(C_{\mathcal{L}}\right)=16\left(\frac{\pi}{\mathcal{L}(\Gamma)}\right)^{4} .
$$

Remark. In this instance, the estimates in Theorem 4 can be obtained more easily by recalling that $A^{\text {st }}(\Gamma)$ is the Friedrichs extension of the symmetric beam operator with continuity of functions and all higher derivatives zero at vertices. The quadratic form domain of all other extensions of this symmetric operator contain the quadratic form domain of $A^{\text {st }}(\Gamma)$. The beam operator $\left(L^{\text {st }}(\Gamma)\right)^{2}$ is one such extension its spectral gap is $\lambda_{2}\left(\left(L^{\text {st }}(\Gamma)\right)^{2}\right)=\left(\lambda_{2}\left(L^{\text {st }}(\Gamma)\right)\right)^{2}$. Since both of these beam operators have zero as their first eigenvalue, it follows that the spectral gap of $A^{\text {st }}(\Gamma)$ is necessarily greater than (or equal to) that of $\left(L^{\text {st }}(\Gamma)\right)^{2}$, whence

$$
\lambda_{2}\left(A^{\mathrm{st}}(\Gamma)\right) \geq\left(\lambda_{2}\left(L^{\mathrm{st}}(\Gamma)\right)\right)^{2} .
$$

The estimates (5.3) and (5.4) are then just a direct corollary of the standard Laplacian estimates (5.1) and (5.2).

Theorem 4 implies that Eulerian graphs have a tendency to have larger spectral gap, which means that their eigenfrequencies are larger. The factor is 16 compared to 4 for Laplacians.

Lemma 5. Let $\mathcal{C}_{2 \mathcal{L}}$ be the loop of length $2 \mathcal{L}$. Then

$$
\lambda_{2}\left(A^{\mathrm{st}}([0, \mathcal{L}])\right)=\lambda_{2}\left(A^{\mathrm{st}}\left(\mathcal{C}_{2 \mathcal{L}}\right)\right)=\left(\frac{\pi}{\mathcal{L}}\right)^{4} .
$$

Proof. Observe that $A^{\text {st }}([0, \mathcal{L}])=\left(L^{\text {st }}([0, \mathcal{L}])\right)^{2}$, so $\lambda_{2}\left(A^{\text {st }}([0, \mathcal{L}])\right)=$ $\left(\frac{\pi}{\mathcal{L}}\right)^{4}$. The same relation with the Laplacian does not hold for $A^{\text {st }}\left(\mathcal{C}_{2 \mathcal{L}}\right)$. However, since $\mathcal{C}_{2 \mathcal{L}}$ is Eulerian, by estimate (5.4) of Theorem 4, we have the lower bound $\lambda_{2}\left(A^{\text {st }}\left(\mathcal{C}_{2 \mathcal{L}}\right)\right) \geq\left(\frac{\pi}{\mathcal{L}}\right)^{4}$. But also $\cos \left(\frac{\pi x}{\mathcal{L}}\right)$ is a nontrivial eigenfunction, so indeed $\lambda_{2}\left(A^{\text {st }}\left(\mathcal{C}_{2 \mathcal{L}}\right)\right)=\left(\frac{\pi}{\mathcal{L}}\right)^{4}$ as claimed.

Finally we prove that the estimate 5.3 is attained if and only if $\Gamma$ is an interval. We argue in a similar manner to the proof of Theorem 3 in [7] for Laplacians. 
Theorem 6. Let $\Gamma$ be a connected finite compact metric graph with total length $\mathcal{L}(\Gamma)$ and let $A^{\mathrm{st}}(\Gamma)$ be the corresponding standard beam operator described by vertex conditions (2.6). If

$$
\lambda_{2}\left(A^{\mathrm{st}}(\Gamma)\right)=\left(\frac{\pi}{\mathcal{L}(\Gamma)}\right)^{4}
$$

then $\Gamma$ is an interval.

Proof. Let $\psi_{2}$ be an eigenfunction of $A^{\text {st }}(\Gamma)$ with eigenvalue $\lambda_{2}\left(A^{\text {st }}(\Gamma)\right)$. It can be extended to a function $\tilde{\psi}_{2}$ on $\Gamma^{2}$ by identifying it with $\psi_{2}$ on the duplicated edges - that is $\left.\tilde{\psi}\right|_{E_{n}}=\left.\tilde{\psi}\right|_{E_{n}^{\prime}}=\left.\psi_{2}\right|_{E_{n}}$ where $E_{n}^{\prime}$ is the duplicate of $E_{n}$ in $\Gamma^{2}$. Then $\tilde{\psi}_{2}$ is an eigenfunction of $A^{\text {st }}\left(\Gamma^{2}\right)$. Now $\tilde{\psi}_{2}$ can be identified as a function from $W_{2}^{4}\left(\mathcal{P}_{2 \mathcal{L}}\right)$ satisfying conditions $(2.5)$ at the vertices. This means in fact that $\tilde{\psi}_{2} \in W_{2}^{2}\left(\mathcal{C}_{2 \mathcal{L}}\right)$. By Lemma 5 ,

$$
\frac{\int_{\mathcal{C}_{2 \mathcal{L}}}\left|\tilde{\psi}_{2}^{\prime \prime}(x)\right|^{2} \mathrm{~d} x}{\int_{\mathcal{C}_{2 \mathcal{L}}}\left|\tilde{\psi}_{2}(x)\right|^{2} \mathrm{~d} x}=\frac{\int_{\Gamma}\left|\psi_{2}^{\prime \prime}(x)\right|^{2} \mathrm{~d} x}{\int_{\Gamma}\left|\psi_{2}(x)\right|^{2} \mathrm{~d} x}=\lambda_{2}\left(A^{\mathrm{st}}\left(\mathcal{C}_{2 \mathcal{L}}\right)\right)
$$

and so $\tilde{\psi}_{2}$ must be the first nontrivial eigenfunction of $A^{\text {st }}\left(\mathcal{C}_{2 \mathcal{L}}\right)$, which by Lemma 5 can, without loss of generality, be identified with $\cos \left(\frac{\pi x}{\mathcal{L}}\right)$.

The original graph $\Gamma$ can be recovered by gluing together pairs of points on $\mathcal{C}_{2 \mathcal{L}}$ where the values of $\tilde{\psi}_{2}$ are equal. But since the preimage of every value in $[-1,1]$ under $\tilde{\psi}_{2}$ consists of exactly two points on $\mathcal{C}_{2 \mathcal{L}}$, the gluing can only possibly done in one way, and $\Gamma$ is an interval of length $\mathcal{L}$ : there could not possibly be any internal vertices since $\cos \left(\frac{\pi x}{\mathcal{L}}\right)$ only satisfies $\psi^{\prime}(x)=0$ at $x=0$ and $x=\mathcal{L}$.

Remark. The final gluing stage of this proof is in contrast with the equivalent proof for quantum graphs where there may be (removable) internal vertices of degree 2 .

This result is a geometric Ambartsumian-type theorem: given only the spectral gap (5.6) and the total length of the graph, one can recover the beam operator (and hence also the graph).

\section{ACKNOWLEDGEMENTS}

The work was supported by the Center for Interdisciplinary Research $(\mathrm{ZiF})$ in Bielefeld in the framework of the cooperation group on 'Discrete and continuous models in the theory of networks' and by the Swedish Research Council grant D0497301. 
We thank I. Popov for attracting our attention to this problem and explaining possible implications for the design of medical tools. 


\section{References}

[1] G. Berkolaiko, J. B. Kennedy, P. Kurasov, and D. Mugnolo, Surgery principles for the spectral analysis of quantum graphs, Trans. Amer. Math. Soc. 372 (2019), no. 7, 5153-5197, DOI 10.1090/tran/7864. MR4009401

[2] M. Sh. Birman and M. Z. Solomjak, Spectral theory of selfadjoint operators in Hilbert space, Mathematics and its Applications (Soviet Series), D. Reidel Publishing Co., Dordrecht, 1987. Translated from the 1980 Russian original by S. Khrushchëv and V. Peller. MR1192782

[3] B. Dekoninck and S. Nicaise, The eigenvalue problem for networks of beams, Linear Algebra Appl. 314 (2000), no. 1-3, 165-189, DOI 10.1016/S0024-3795(00)00118-X. MR1769018

[4] L. Friedlander, Extremal properties of eigenvalues for a metric graph, Annales de linstitut Fourier 55 (2005), DOI 10.5802/aif.2095.

[5] F. Gregorio and D. Mugnolo, Bi-Laplacians on graphs and networks, D. J. Evol. Equ., posted on 11 Jul. 2019, DOI 8-019-00523-7.

[6] J.-C. Kiik, P. Kurasov, and M. Usman, On vertex conditions for elastic systems, Phys. Lett. A 379 (2015), no. 34-35, 1871-1876, DOI 10.1016/j.physleta.2015.05.017. MR3349572

[7] P. Kurasov and S. Naboko, Rayleigh estimates for differential operators on graphs, J. Spectr. Theory 4 (2014), no. 2, 211-219, DOI 10.4171/JST/67. MR3232809

[8] P. Kurasov and J. Muller, Higher order differential operators on graphs and almost periodic functions. To appear.

[9] L. D. Landau and E. M. Lifshitz, Theory of elasticity, Course of Theoretical Physics, Vol. 7. Translated by J. B. Sykes and W. H. Reid, Pergamon Press, London-ParisFrankfurt; Addison-Wesley Publishing Co., Inc., Reading, Mass., 1959. MR0106584 